\title{
Eye movement desensitisation and reprocessing therapy $v$. stabilisation as usual for refugees: randomised controlled trial
}

\author{
F. Jackie June ter Heide, Trudy M. Mooren, Rens van de Schoot, Ad de Jongh and Rolf J. Kleber
}

\section{Background}

Eye movement desensitisation and reprocessing (EMDR) therapy is a first-line treatment for adults with post-traumatic stress disorder (PTSD). Some clinicians argue that with refugees, directly targeting traumatic memories through EMDR may be harmful or ineffective.

\begin{abstract}
Aims
To determine the safety and efficacy of EMDR in adult refugees with PTSD (trial registration: ISRCTN20310201).
\end{abstract}

\section{Method}

In total, 72 refugees referred for specialised treatment were randomly assigned to $12 \mathrm{~h}$ of EMDR $(3 \times 60$ min planning/ preparation followed by $6 \times 90$ min desensitisation/ reprocessing) or $12 \mathrm{~h}(12 \times 60 \mathrm{~min})$ of stabilisation. The Clinician-Administered PTSD Scale (CAPS) and Harvard Trauma Questionnaire (HTQ) were primary outcome measures.

\section{Results}

Intention-to-treat analyses found no differences in safety (one severe adverse event in the stabilisation condition only) or efficacy (effect sizes: CAPS -0.04 and HTQ 0.20) between the two conditions.

\section{Conclusions}

Directly targeting traumatic memories through $12 \mathrm{~h}$ of EMDR in refugee patients needing specialised treatment is safe, but is only of limited efficacy.

\section{Declaration of interest}

A.d.J. reports receiving personal fees from teaching activities and from books about trauma and its treatment (including EMDR). He is a board member of the Dutch EMDR Association and the EMDR Europe Association.

\section{Copyright and usage}

(c) The Royal College of Psychiatrists 2016.
Experiences of war and organised violence in combination with post-migration stressors ${ }^{1}$ leave asylum seekers and refugees in Western countries at relatively high risk of developing posttraumatic stress disorder (PTSD)., ${ }^{2,3}$ PTSD in adult refugees has been shown to pose a burden not only for individuals and their families, ${ }^{4}$ but also for communities at large. ${ }^{5}$ Psychological treatment for refugees with chronic PTSD, although imperative, is a great clinical challenge. ${ }^{6}$ According to evidence-based guidelines for the treatment of adults with chronic PTSD, trauma-focused cognitive-behavioural therapy (TFCBT) and eye movement desensitisation and reprocessing (EMDR) therapy should be offered to all patients with this disorder. ${ }^{7,8}$ With refugee patients the experience-based recommendation is often made that trauma-focused treatment should be preceded or even replaced by stabilisation., ${ }^{7,9}$ Trauma-focused therapy per se, especially for refugees living in unstable conditions, has been suggested to cause unmanageable distress ${ }^{10}$ and to be inappropriate and ineffective. ${ }^{7}$ Nevertheless, systematic reviews of the psychological treatment of refugees have shown TFCBT and narrative exposure therapy (NET) to be safe and efficacious with refugees in various social conditions. ${ }^{10-12}$ However, no full, high-quality randomised trials of EMDR therapy with refugees have yet been conducted. To determine the safety and efficacy of EMDR therapy in traumatised refugees, we designed a trial in which adult asylum seekers and refugees with chronic PTSD were randomly assigned to either EMDR therapy or stabilisation. In line with a pilot study ${ }^{13}$ and evidence-based guidelines, our first hypothesis was that EMDR therapy would not differ from stabilisation in the occurrence of harms (defined as symptom increase and drop-out related to symptom increase). Our second hypothesis was that EMDR therapy would be more efficacious than stabilisation in reducing trauma-related symptoms (PTSD, anxiety and depression) and improving quality of life.

\section{Method}

The trial was performed at Foundation Centrum '45, a highly specialised Dutch centre for diagnostics and treatment of psychotrauma resulting from persecution, war and violence. Centrum ' 45 receives national referrals of patients considered too complex to be treated in their own municipalities. Participants were enrolled at two out-patient teams for refugees (in the towns of Oegstgeest and Diemen). Patients judged eligible for participation were asked by their intake therapists if they wished to receive any information about the study and, upon consent, were informed about the study by a research associate. Both treatments were presented as aimed at diminishing PTSD symptoms: EMDR through desensitisation of traumatic memories, stabilisation through enhancement of coping with PTSD symptoms and stressful circumstances in the here-andnow. Those willing to participate signed an informed consent form and were then interviewed with the MINI International Neuropsychiatric Interview (MINI) ${ }^{14}$ to formally check inclusion and exclusion criteria. Data collection took place from September 2009 until August 2012. The trial was approved by the medical ethics committee of the University of Leiden. Trial registration: NARCIS (Dutch National Academic Research and Collaborations Information System) OND1324839; ISRCTN20310201.

\section{Study entry criteria}

Refugees who applied for treatment at Centrum ' 45 were eligible for participation if they were at least 18 years of age, met the criteria for a PTSD diagnosis according to the DSM-IV-TR, ${ }^{2}$ and asked for individual therapy to diminish their PTSD symptoms. Patients who had at some point claimed asylum in 
The Netherlands - irrespective of whether their claim had been met or rejected or was still under consideration - were defined as 'refugee'. Patients were excluded if they had disorders that acutely threatened their mental or physical health (i.e. depression with high suicidal intent or psychotic features, psychotic disorder, bipolar disorder and severe self-harm or eating disorders) or that interfered with their ability to participate (i.e. alcohol or substance dependence and cognitive disorders). No restrictions were placed on either refugee status or language proficiency. No other psychotherapeutic treatment could take place during the study, and psychotropic medication had to be kept stable from 2 months before treatment until the post-treatment assessment. For those participants who developed high suicidal intent, a psychotic disorder or another serious psychiatric disorder during the study, a psychiatric consultation was prescribed during which the necessity of prescribing or changing psychotropic medication (using a medication protocol) and of discontinuation of the intervention were to be evaluated.

\section{Trial design}

A two-arm design was used in which participants were randomly assigned to either $12 \mathrm{~h}$ ( 9 sessions) of EMDR therapy or $12 \mathrm{~h}(12$ sessions) of stabilisation as usual. To create maximum ecological validity, the recommended session length of EMDR and stabilisation was preserved, ${ }^{7}$ and conditions were equated on number of treatment hours rather than number of sessions. Twelve treatment hours is considered a minimum to reach improvement in multiply traumatised patients. ${ }^{7}$

Blocked, simple randomisation was conducted with the latest two participants who had satisfied the inclusion criteria at the same study site forming a block. Participants were assigned to their experimental group through flipping a coin: the outcome (EMDR therapy for heads, stabilisation for tails) was assigned to the participant lowest in the alphabet. An independent research associate who was not otherwise involved in the inclusion process performed randomisation. As both the EMDR condition and the stabilisation condition contained active treatment elements, the design was complemented with a naturalistic waitlist condition to control for time (see online supplement DS1). Primary outcome measures consisted of the change in PTSD symptom severity and diagnosis, both clinician-rated (Clinician-Administered PTSD Scale, CAPS) ${ }^{15}$ and self-administered (Harvard Trauma Questionnaire, HTQ). ${ }^{16}$ Secondary outcome measures were changes in anxiety and depression (Hopkins Symptom Checklist, HSCL-25) ${ }^{17}$ and quality of life (World Health Organization Quality of Life Assessment, WHOQOL-BREF). ${ }^{18}$ Harms were defined as an increase in PTSD symptoms of at least ten points on the CAPS ${ }^{19}$ and premature termination of the study treatment because of symptom increase. All instruments were administered before treatment $\left(T_{1}\right), 2$ weeks post-treatment $\left(T_{2}\right)$ and at a 3-month follow-up $\left(T_{3}\right)$. After $T_{2}$, participants converted to care as usual.

\section{Interventions}

EMDR therapy is a trauma-focused treatment that consists of several steps including treatment planning, preparing the patient for trauma-focused treatment, desensitisation and reprocessing, and evaluation. During desensitisation and reprocessing (which is considered the main active element) a focus on a traumatic image and the thoughts, sensations, feelings and memories that it elicits, is combined with an attention-demanding task such as tracking the therapist's fingers with the eyes. ${ }^{20}$ The EMDR condition started with three $60 \mathrm{~min}$ sessions dedicated to treatment planning and preparation (including discussing study course and use of interpreters, discussing the patient's explanatory model and subsequently providing psychoeducation on PTSD and EMDR, and making a timeline of traumatic experiences and symptoms). Traumatic memories that were expected to lead to the greatest remission of PTSD symptoms were selected for desensitisation. The preparatory sessions were followed by six 90 min desensitisation sessions, using the Dutch version of the EMDR protocol. ${ }^{21}$ Stabilising interventions were proscribed. The EMDR condition was performed by seven clinical psychologists, one physician/psychotherapist and two psychotherapists. Only therapists who had earlier in their career completed an accredited advanced EMDR course participated. Their average experience in EMDR therapy was 5.3 years $($ s.d. $=2.9$ ). They received monthly supervision by a licensed EMDR supervisor.

The stabilisation condition consisted of 12 sessions of $60 \mathrm{~min}$ stabilisation as usual. In phase-oriented treatment for PTSD, the first phase or stabilisation phase is aimed at enhancing safety, control over symptoms and socio-psychological competencies through interventions such as emotion regulation and relational skills building, stress management and cognitive restructuring; processing of traumatic memories is left until the second phase. ${ }^{9}$ Stabilisation as usual, rather than a structured form of stabilisation, was chosen as a control condition to reflect the regular non-structured stabilisation offered in European mental healthcare centres for refugees. ${ }^{22,23}$ Therapists were asked to select stabilising interventions to match their patient's needs. Exposure to traumatic memories was proscribed. Stabilisation was performed by three clinical psychologists, five psychotherapists, one physician/systemic therapist, one psychiatrist, one social psychiatric nurse, two psychiatrists in training and one psychotherapist in training. Those therapists participated who regularly conducted stabilising interventions in their usual care. Their mean number of years of experience in working with traumatised refugees was 9.9 (s.d.=5.5), which did not differ from EMDR therapists' experience (mean 9.00, s.d. =5.5; $t(22)=-0.41, P=0.75)$. Stabilisation therapists received monthly supervision from a registered cognitive-behavioural and family therapy supervisor/trainer with a specialisation in trauma therapy.

For both conditions, therapist manuals were designed with information on the study methods (such as study design and rules for drop-out), study treatment (such as pre- and proscribed elements), the medication protocol and camera use (manuals available from the authors on request). To assess treatment integrity, treatment sessions were videotaped. For the EMDR condition, a detailed treatment fidelity scale was put together consisting of the scale used in a Dutch EMDR trial ${ }^{24}$ and additional prescribed, proscribed and non-specific elements. For the stabilisation condition, a brief treatment fidelity scale was designed containing prescribed, proscribed and non-specific elements. All interventions were delivered in Dutch when possible and translated by registered interpreters (physically present or by telephone) when necessary.

\section{Measures}

PTSD severity and diagnosis were measured by the CAPS and the HTQ. The CAPS yields frequency and intensity scores (ranging from 0 to 4) for all PTSD symptoms according to the DSM-IV-TR in the past week. A symptom was considered present if its frequency was rated as at least 1 and its intensity as at least $2 .^{25}$ To capture full PTSD severity, interviewers referred to clusters of war or persecution experiences rather than to one index traumatic event. The HTQ consists of three parts: one on traumatic events, one on DSM-IV trauma symptoms, and one on other trauma symptoms. Scores for the symptom parts range from 1 (not at 
all) to 4 (extremely). Anxiety and depression were measured using the HSCL-25, which uses the same scale. Quality of life was measured by the WHOQOL-BREF, which measures four domains of quality of life (physical, psychological, social relationships, and environment) on a scale of 1-5 (a higher score indicating a higher quality of life). All of these instruments have good psychometric properties and are widely used in transcultural research. ${ }^{26-29}$

Interpreters were used whenever the participant did not speak Dutch and the instrument was not available in the participant's native language. To maximise participant understanding of the questionnaires while minimising dependency on individual interpreters' skills, where possible we used questionnaires in the participant's native language that had been carefully translated by our institute (HTQ, HSCL) ${ }^{30}$ or by the WHOQOL Group (WHOQOL-BREF) ${ }^{18}$ Interviews were administered by trained Master's students in psychology who were kept masked to treatment condition by having limited access to participant data and by asking participants not to reveal treatment content. They received monthly supervisions of their CAPS ratings using videotaped interviews. Participants received a gift coupon at $T_{2}$ and a box of chocolates at $T_{3}$.

\section{Statistical analysis}

Sample size was calculated with the power analysis program $\mathrm{G}^{\star}$ Power version 2 for Windows (Erdfelder, Faul and Buchner at the University of Trier, Germany, www.psycho.uni-duesseldorf.de/ aap/projects/gpower/). Power calculations were based on outcomes of our pilot study, which resulted in a medium effect size between EMDR and stabilisation on the HTQ. ${ }^{13}$ For the main study, a sample size of 36 patients per condition was needed (using a power of 0.80, a two-sided significance level of 0.05 and three repeated measures) to detect a medium between-treatment effect size at $T_{3}$. In response to reviews of our pilot study, in the final analyses we used a statistically more advanced variation of the planned analysis strategy (Bayesian latent growth modelling instead of repeated measures analysis), which the sample size allowed for. $^{31}$

Treatment fidelity, interrater reliability and demographic and clinical variables were analysed with SPSS version 20.0 for Windows. Chi-squared and $t$-tests were conducted to check for demographical and clinical differences between participants and those who refused to participate as well as between the two treatment conditions. Mean scale and subscale scores were computed, allowing for a maximum number of three missing values in the HTQ and HSCL and following the questionnaire manual rules for missing values for the WHOQOL-BREF. Chi-squared tests (for treatment condition, gender, refugee status, drop-out, use of an interpreter and work status) and $t$-tests (for age, distance from home to treatment centre, and CAPS score at baseline) were conducted to explore relationships between missing values and demographic and clinical variables.

Data were then converted to Mplus version 7 (Muthén and Muthén at University of California, www.statmodel.com/). Bayesian estimation was used in all analyses with the default settings in Mplus with regard to prior specifications. ${ }^{32}$ Bayesian analysis enables full intent-to-treat analysis as missing data are automatically imputed. For the burn-in and convergence criteria we used a minimum of 20000 iterations after which the Gelman-Rubin convergence criterion $^{32}$ was used to monitor convergence with a cut-off value of 0.01 . Additionally, to ensure convergence was reached, we checked the trace-plots manually. In Bayesian statistics, credibility intervals are used to indicate the $95 \%$ probability that the estimate will lie between the lower and upper value of the interval. A treatment effect can be assumed to be present when the credibility interval does not include zero. A difference in treatment effect between conditions can be assumed to be present when credibility intervals between conditions do not overlap. As a measure of effect size between conditions we divided the difference between the linear slopes by the square root of the linear slope's variance (which is equal for the two conditions). ${ }^{33}$

Finally, to analyse individual changes in post-traumatic stress scores over time and to identify predictors in the separate treatment conditions, we applied a multigroup latent growth model to the data. Latent growth modelling (LGM) enables an examination of individual growth trajectories for each condition, allowing participants to have a different starting point (i.e. a random intercept model) and a different growth rate (i.e. a random slope model). The $\mathrm{R}$-squared statistic provides the proportion of variance in post-traumatic stress symptoms that is explained by the latent growth factors. The posterior predictive $P$-value was checked as an indication for model fit (outcome files available from the authors on request).

\section{Results}

\section{Participants}

Participant flow during the trial is depicted in Fig. 1. The flow diagram includes all patients who met inclusion criteria at intake, i.e. before they were informed about the study and formally interviewed. ${ }^{34}$ Although the inclusion of 72 participants was planned, 2 participants who terminated their participation before the first treatment session, unaware of which condition they had been assigned to, were replaced as it was clear that no post-treatment data could be obtained for them.

Chi-squared and $t$-tests revealed no significant demographic or clinical differences between participants and those who refused to participate (data available from the authors on request). Table 1 shows demographic and clinical characteristics for the two groups at baseline (see online Table DS1 for a version of this table that details a wider range of demographic characteristics). The EMDR group was found to contain significantly fewer female participants than the stabilisation group. Types of traumatic experiences most frequently reported in the HTQ were being close to death (60/72, $83 \%)$, murder of family or friend $(54 / 72,75 \%)$ and threatened with torture $(52 / 72,72 \%)$. Drop-out numbers for the two groups were comparable, with 6 EMDR participants (16.7\%) and 8 stabilisation participants $(22.2 \%)$ discontinuing the intervention $\left(\chi^{2}(1, n=72)=0.36, P=0.55\right)$. One participant, in the stabilisation group, terminated treatment prematurely because of symptom increase (attempted suicide). In both groups, asylum seekers and those in the country illegally were no more likely to drop out of treatment than participants with a refugee status (EMDR therapy: $\chi^{2}(1, n=36)=0.05, \quad P=0.83 ; \quad$ stabilisation: $\quad \chi^{2}(1, n=36)=0.05$, $P=0.83)$.

\section{Treatment integrity and content}

Treatment integrity was rated by four trained graduate-level research assistants. We randomly selected $12.5 \%$ of all treatment sessions for rating of treatment fidelity. Interrater agreement was determined for the first three ratings for both conditions. As this was consistently high (Cronbach's $\alpha=0.95$ for EMDR and 1.0 for stabilisation), interrater agreement was deemed to be satisfactory and was not monitored thereafter.

For the EMDR group, 36 out of 291 sessions were rated, a third of which were taken from the preliminary sessions and two-thirds of which from the EMDR protocol sessions. The mean treatment fidelity score for the preliminary sessions was 97.0 (s.d.=3.0) and for the protocol sessions $87.8($ s.d. $=9.2)$ on a scale of $0-100$. For EMDR treatment completers $(n=30)$, subjective 


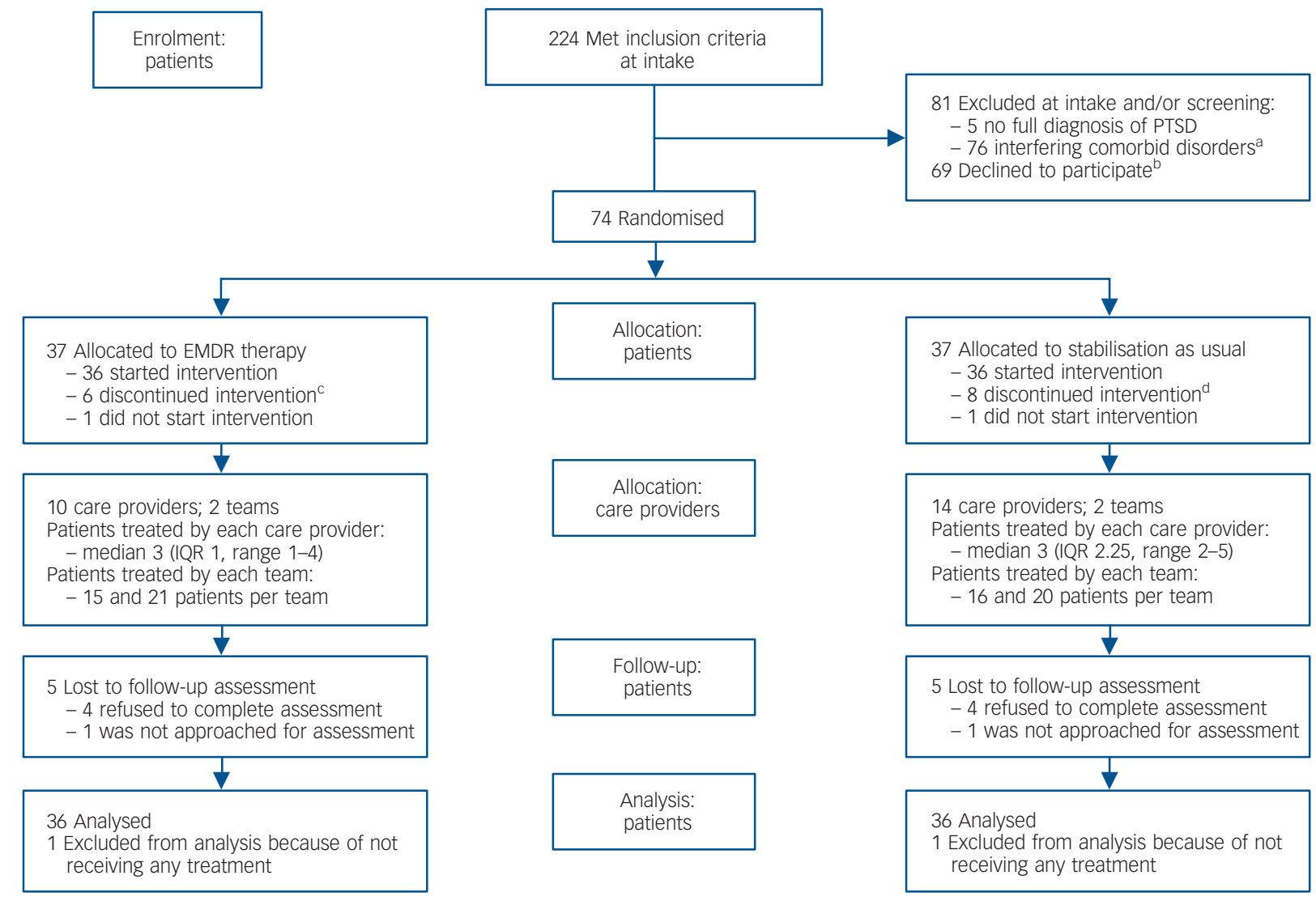

Fig. 1 CONSORT flow diagram.

EMDR, eye movement desensitisation and reprocessing; IQR, interquartile range.

a. 34 substance or alcohol dependence, 7 self-harm, 3 cognitive disorder, 5 eating disorder, 10 serious suicidal ideations, 16 psychotic disorder, 1 bipolar disorder.

b. 10 did not want any help at the institute, 30 found participation too much hassle, 18 did not want trauma-focused treatment, 3 did not want stabilisation, 2 did not want treatment for post-traumatic stress disorder (PTSD), 6 refused for various study-related reasons.

c. 3 did not show up for 4 consecutive appointments; 1 thought the travel distance too great; 2 did not want to continue trauma-focused therapy.

d. 6 did not show up for 4 consecutive appointments; 1 developed high suicidal intent; 1 wanted to change to trauma-focused therapy.

unit of distress (SUD) scores decreased significantly from the start of treatment to the end of treatment (from a mean of 8.3, s.d. $=1.7$ to a mean of 3.9 , s.d. $=3.7, t(29)=7.5, P<0.001$ ), with only 11 participants reaching the desired SUD of $0-1$. Mean number of targets treated was 1.6 (s.d. $=1.0$, range $1-5$ ), with most participants $(19 / 30,63 \%)$ staying with one target.

For the stabilisation group, 48 out of 387 sessions were rated. The mean treatment fidelity for the stabilisation condition was 88.7 (s.d. $=8.9$ ) on a scale of $0-100$. For these 48 sessions the main interventions were registered using an intervention menu. ${ }^{13}$ The most frequently registered interventions were discussing and teaching of coping strategies, identification and validation of negative emotions, and active problem-solving by participant and therapist.

For three EMDR participants and three stabilisation participants a change of medication took place during the study. In most cases this entailed a new or changed prescription for antidepressants.

\section{Reliability}

To assess interrater reliability, $12.5 \%$ of all interviews (MINI and CAPS) were randomly selected, using stratification for time of assessment. Interrater reliability for the decision whether or not to include a patient in the study (using the MINI; 12.5\%, 12/94) was

\begin{tabular}{|c|c|c|c|c|c|}
\hline & $\begin{array}{l}\text { EMDR group } \\
\quad(n=36)\end{array}$ & $\begin{array}{l}\text { Stabilisation group } \\
\qquad(n=36)\end{array}$ & $\chi^{2}$ (d.f.) & $t$-test (d.f) & $P$ \\
\hline Age, years: mean (s.d.) & $43.1(10.7)$ & $39.8(11.9)$ & & $1.26(70)$ & 0.21 \\
\hline Women, $n(\%)$ & $6(16.7)$ & $14(38.9)$ & $4.43(1)$ & & 0.04 \\
\hline Types of traumatic experiences, HTQ: mean (s.d.) & $13.8(5.5)$ & $13.7(5.6)$ & & $0.85(70)$ & 0.93 \\
\hline Years with PTSD, mean (s.d.) & $7.9(7.2)$ & $8.0(6.5)$ & & $-0.41(59)$ & 0.97 \\
\hline Comorbid depression, $n$ (\%) & $28(77.8)$ & $28(77.8)$ & $0.00(1)$ & & 1.00 \\
\hline On psychotropic medication, $n$ (\%) & $21(58.3)$ & $21(58.3)$ & $0.00(1)$ & & 1.00 \\
\hline CAPS symptom severity, mean (s.d.) & $74.7(18.0)$ & $78.3(18.3)$ & & $-0.83(70)$ & 0.41 \\
\hline Use of interpreter during study treatment, $n$ (\%) & $20(55.6)$ & $20(55.6)$ & $0.00(1)$ & & 1.00 \\
\hline
\end{tabular}


excellent (a Cronbach's $\alpha$ of 1 ). Interrater reliability for the CAPS $(12.5 \%, 25 / 198)$ was excellent for PTSD symptom severity (Cronbach's $\alpha=0.95$ ) and good for PTSD diagnosis (Cohen's $\kappa=0.78$ ). Internal consistency for all scales was excellent, with a Cronbach's $\alpha$ of 0.86 for the CAPS; 0.88 for HTQ symptoms; 0.90 for the HSCL; and 0.85 for the WHOQOL-BREF.

\section{Missing data}

Out of a total database of 1944 total or mean scores (i.e. nine outcome measures administered three times with 72 participants), 186 scores $(9.6 \%)$ were missing and automatically imputed for each Bayesian analysis. 'Missingness' was significantly related to drop-out, with those who ended participation prematurely being more likely to have missing data than those who completed the study $\left(\chi^{2}(1, n=72)=12.85, P<0.001\right)$.

\section{Outcomes}

\section{Primary outcomes}

Table 2 describes PTSD diagnoses for the two groups at each assessment.

Numbers indicate that outcomes displayed a slightly quadratic development over time for both groups. Between $T_{1}$ and $T_{3}$, the majority of assessment completers in both groups achieved a clinically significant improvement in PTSD severity (defined as improving at least ten points on the CAPS, Table 2). ${ }^{19}$

In the LGM analyses, best model fit was obtained when including a quadratic slope. Table 3 shows the results of the intent-to-treat analyses for primary outcomes. Participants in both groups initially achieved a clinically significant improvement in clinician-rated PTSD severity, which was partly lost after $T_{2}$. The EMDR group significantly improved in self-reported PTSD symptoms according to DSM-IV. No significant differences between the two groups were found in either linear or quadratic slopes and effect sizes between the groups were small (for withinand between-treatment effect sizes based on unimputed data, see online Table DS2).

\section{Secondary outcomes}

No significant differences were found between the EMDR therapy and stabilisation group on any of the secondary outcome measures (Table 3). Neither intervention had a significant effect on anxiety, depression or quality of life. Quality of life, in fact, in both groups did not show uniform improvement.

\section{Post hoc analyses}

\section{Gender}

As the EMDR group contained significantly fewer female participants than the stabilisation group, we added gender as a covariate to the
LGM model. This, however, led to a decreased model fit. We therefore analysed the effect of gender on the slopes of the primary outcome measures. Low R squares for all measures and conditions showed that gender had little influence on treatment effect (online Table DS3).

\section{Refugee status}

As some clinicians argue that EMDR therapy with asylum seekers is not possible because of their insecure living conditions, we also analysed the effect of refugee status on the primary outcome measures. We divided the groups into participants with no refugee status (i.e. asylum seekers and those staying in the country illegally) and those with temporary or permanent refugee status. The direction of the effect was that participants without a refugee status, regardless of treatment group, showed more PTSD symptom reduction than participants with a refugee status. Although not statistically significant, in the stabilisation group the effect size was medium (online Table DS3).

\section{Discussion}

\section{Main findings}

In this study, no differences in safety or efficacy were found between EMDR therapy and stabilisation as usual. As previously stated, some clinicians argue that trauma-focused treatment in refugees, especially those living in unstable circumstances, may be harmful. ${ }^{10}$ However, in this study, the EMDR and stabilisation groups had comparable numbers of participants who dropped out of treatment and participants reporting symptom increase. In fact, drop-out numbers were relatively low compared with other PTSD outcome studies. ${ }^{36}$ Additionally, in the EMDR as well as the stabilisation group asylum seekers showed an improvement at least equal to that of refugees. Results are in line with an increasing body of evidence suggesting that trauma-focused therapy carries no risk of psychologically overwhelming refugee patients, even those in unstable conditions. ${ }^{10}$ However, conclusions may not generalise to refugee patients who meet our exclusion criteria notably those with psychotic disorders, substance dependence or severe suicidal ideations - although the justifiability of using these exclusion criteria in patients who are treated for these disorders has been called into question. ${ }^{37}$

Comparison with a non-randomised waitlist condition suggested that treating refugees with EMDR therapy is more effective than not treating them. However, contrary to expectation, EMDR therapy was found to be no more effective than stabilisation. The effect of stabilisation was similar to effects found for unstructured stabilisation in other refugee samples in Western countries. ${ }^{22,23}$ However, the effect of EMDR therapy was lower than expected, with effect sizes for other trauma-focused therapies

\begin{tabular}{|c|c|c|c|c|}
\hline & \multicolumn{2}{|c|}{$n / N(\%)$} & \multirow[b]{2}{*}{$\chi^{2}$ (d.f.) } & \multirow[b]{2}{*}{$P$} \\
\hline & EMDR group & Stabilisation group & & \\
\hline CAPS diagnosis & $30 / 36(83)$ & $32 / 36(89)$ & & \\
\hline$T_{1}$ & $21 / 33(64)$ & $20 / 29(69)$ & $0.47(1)$ & 0.50 \\
\hline$T_{2}$ & $26 / 32(81)$ & $22 / 31(71)$ & $0.08(1)$ & 0.78 \\
\hline$T_{3}$ & & & $0.92(1)$ & 0.34 \\
\hline CAPS severity change $T_{1}-T_{3}$ & & & $0.23(2)$ & 0.89 \\
\hline Deterioration ( $\geqslant-10$ points) & 7/32 (21.9) & 8/31 (25.8) & & \\
\hline No change ( $<10$ points to $>-10$ points) & $12 / 32(37.5)$ & 10/31 (32.3) & & \\
\hline Improvement ( $\geqslant 10$ points) & $13 / 32(40.6)$ & $13 / 31(41.9)$ & & \\
\hline
\end{tabular}




\begin{tabular}{|c|c|c|c|c|c|c|c|c|c|}
\hline & \multirow[b]{2}{*}{ Intercept } & \multirow[b]{2}{*}{ Slope } & \multicolumn{2}{|c|}{ Credibility interval } & \multirow[b]{2}{*}{ Effect size ${ }^{a}$} & \multirow[b]{2}{*}{ Q slope } & \multicolumn{2}{|c|}{ Credibility interval } & \multirow[b]{2}{*}{ Effect size ${ }^{a}$} \\
\hline & & & Lower $2.5 \%$ & Upper $2.5 \%$ & & & Lower $2.5 \%$ & Upper $2.5 \%$ & \\
\hline \multicolumn{10}{|l|}{ Primary outcomes } \\
\hline \multicolumn{10}{|l|}{ CAPS, severity } \\
\hline EMDR therapy group & 74.71 & -11.68 & -24.91 & 1.12 & \multirow[t]{2}{*}{-0.04} & 4.66 & -1.70 & 10.50 & \multirow[t]{2}{*}{-0.03} \\
\hline Stabilisation group & 78.30 & -12.75 & -27.26 & 1.61 & & 4.33 & -2.55 & 10.98 & \\
\hline \multicolumn{10}{|l|}{ HTQ, DSM-IV } \\
\hline EMDR therapy group & 3.06 & $-0.40^{\star}$ & -0.74 & -0.04 & \multirow[t]{2}{*}{0.20} & 0.15 & -0.01 & 0.30 & \multirow[t]{2}{*}{-0.29} \\
\hline Stabilisation group & 3.19 & -0.25 & -0.57 & 0.06 & & 0.06 & -0.08 & 0.20 & \\
\hline \multicolumn{10}{|l|}{ HTQ, total } \\
\hline EMDR therapy group & 2.85 & -0.31 & -0.63 & 0.02 & \multirow[t]{2}{*}{0.29} & 0.12 & -0.02 & 0.27 & \multirow[t]{2}{*}{-0.38} \\
\hline Stabilisation group & 2.90 & -0.11 & -0.41 & 0.18 & & 0.02 & -0.11 & 0.14 & \\
\hline \multicolumn{10}{|l|}{ Secondary outcomes } \\
\hline \multicolumn{10}{|l|}{$\mathrm{HSCL}$, anxiety } \\
\hline EMDR therapy group & 2.86 & -0.09 & -0.43 & 0.25 & \multirow[t]{2}{*}{0.09} & 0.02 & -0.13 & 0.16 & \multirow[t]{2}{*}{-0.15} \\
\hline Stabilisation group & 3.04 & -0.01 & -0.45 & 0.43 & & -0.03 & -0.23 & 0.17 & \\
\hline \multicolumn{10}{|l|}{ HSCL, depression } \\
\hline EMDR therapy group & 2.95 & -0.20 & -0.55 & 0.16 & \multirow[t]{2}{*}{-0.03} & 0.06 & -0.09 & 0.21 & \multirow[t]{2}{*}{0.03} \\
\hline Stabilisation group & 2.97 & -0.22 & -0.59 & 0.18 & & 0.07 & -0.09 & 0.23 & \\
\hline \multicolumn{10}{|l|}{ WHOQOL-BREF, physical } \\
\hline EMDR therapy group & 2.35 & 0.07 & -0.36 & 0.49 & \multirow[t]{2}{*}{0.07} & -0.03 & -0.23 & 0.16 & \multirow[t]{2}{*}{-0.10} \\
\hline Stabilisation group & 2.34 & -0.01 & -0.44 & 0.43 & & 0.01 & -0.18 & 0.21 & \\
\hline \multicolumn{10}{|c|}{ WHOQOL-BREF, psychological } \\
\hline EMDR therapy group & 2.34 & 0.00 & -0.47 & 0.47 & \multirow[t]{2}{*}{0.07} & -0.03 & -0.22 & 0.17 & -0.15 \\
\hline Stabilisation group & 2.35 & -0.09 & -0.61 & 0.44 & & 0.04 & -0.19 & 0.27 & \\
\hline WHOQOL-BREF, social rel & & & & & & & & & \\
\hline EMDR therapy group & 2.71 & 0.05 & -0.48 & 0.58 & -0.28 & -0.09 & -0.33 & 0.15 & 0.22 \\
\hline Stabilisation group & 2.56 & 0.38 & -0.18 & 0.95 & & -0.20 & -0.46 & 0.05 & \\
\hline WHOQOL-BREF, environm & & & & & & & & & \\
\hline EMDR therapy group & 2.99 & -0.24 & -0.61 & 0.14 & -0.52 & 0.06 & -0.11 & 0.24 & 0.38 \\
\hline Stabilisation group & 2.68 & 0.22 & -0.17 & 0.63 & & -0.08 & -0.26 & 0.10 & \\
\hline
\end{tabular}

in refugee samples in Western countries ranging from 0.93 to 1.6 for $\mathrm{NET}^{22,23}$ and from 2.4 to 2.6 for exposure. ${ }^{10,38}$ A primary explanation is that the number of trauma-focused sessions was lower in this study (i.e. 6 sessions of desensitisation and reprocessing) than in comparable studies ( 9 for $\mathrm{NET}^{22,23}$ and 20 for $\mathrm{TFCBT}^{38}$ ). Considering the high number of types of traumatic experiences (14 in both conditions), six sessions appear to have been insufficient to process all memories driving PTSD symptom severity. Additionally, it is possible that equalisation of number of treatment sessions rather than number of treatment hours would have resulted in differences in efficacy between the two groups, as it did in our pilot study. ${ }^{13}$ A second explanation is that the study sample consisted of refugee patients who are relatively difficult to treat. Centrum ' 45 is a specialised institute that receives national referrals of patients who have insufficiently benefited from, or are expected to insufficiently benefit from, treatment within primary mental healthcare. This may be related to the complexity of their traumatic experiences (i.e. multiple, prolonged, interpersonal traumatic events often involving intentional and extreme cruelty) as well as the complexity of their present-day lives (such as being threatened with expulsion, having no financial means, being socially isolated, fearing the effects of ongoing conflict in the country of origin). ${ }^{39}$ Comparable studies included only participants who were fluent in the language of their resettlement country $^{38}$ or who could be treated within general healthcare. ${ }^{23} \mathrm{~A}$ third possible explanation is that TFCBT (including NET) is indeed more effective than EMDR therapy in treating refugees with chronic PTSD. The culturally sensitive rationale ${ }^{40}$ and relatively simple protocol of NET might make this therapy easier to grasp for refugees and EMDR therapy may have some catching up to do in those respects. A randomised controlled trial is needed to clarify this issue.

\section{Strengths and limitations}

This study is the first full trial that meets all CONSORT criteria to test the safety and efficacy of EMDR therapy in refugees with chronic PTSD. A broad range of refugee patients were engaged in this study, including patients who needed interpreters and highly vulnerable patients who were homeless, stayed in the country illegally or were listed for forced return. Both groups were treated by highly experienced therapists. Bayesian estimation allowed for full intention-to-treat analysis.

The study also has several limitations. Although all instruments used have been extensively validated in refugee or transcultural samples, not all language versions were validated, which may have compromised measurement validity. In addition, a measure of positive expectancy for both therapists and patients would have been useful to explore the influence of treatment preference on treatment outcome. Asking refugees which treatment would be preferable under which circumstances would have yielded valuable information and would have enabled refugees to contribute as experts as well as participants. Finally, inequality in number of treatment sessions between the two groups may have led to a greater risk of treatment drop-out in the stabilisation condition than in the EMDR condition.

\section{Clinical implications}

On the basis of our study it may be concluded that therapists need not refrain from offering EMDR therapy to asylum seekers or 
refugees with chronic PTSD for fear of deterioration, although this conclusion may not necessarily generalise to refugees with comorbid untreated psychosis, substance dependence and high suicidal intent. Offering only a limited number of EMDR sessions, as was the case in this study, may not result in a satisfactory reduction of PTSD and comorbid symptoms. Whether EMDR therapy would show greater efficacy with refugees after a larger number of sessions, or with refugee patients who are referred to general rather than specialised mental healthcare, or when preceded by or combined with stabilising interventions, remains to be tested in future trials. This study adds to an increasing body of evidence that directly targeting traumatic memories of refugees carries no harm.

F. Jackie June ter Heide, PhD, MPhil (Cantab), Trudy M. Mooren, PhD, Foundation Centrum ' 45 - partner in Arq Psychotrauma Expert Group, Oegstgeest and Diemen, The Netherlands; Rens van de Schoot, PhD, Department of Methods and Statistics, Utrecht University, Utrecht, The Netherlands and Optentia Research Program, Faculty of Humanities, North-West University, Vanderbijpark, South Africa; Ad de Jongh, PhD, Department of Behavioural Sciences, Academic Centre for Dentistry Amsterdam, University of Amsterdam, Vrije University, Amsterdam, The Netherlands, and School of Health Sciences, Salford University, Manchester, UK; Rolf J. Kleber, PhD, Foundation Centrum ' 45 - partner in Arq Psychotrauma Expert Group, Diemen and Department of Clinical \& Health Psychology, Utrecht University, Utrecht, The Netherlands

Correspondence: F. J. J. ter Heide, PhD, MPhil (Cantab), Foundation Centrum'45 - partner in Arq Psychotrauma Expert Group, Nienoord 5, 1112 XE Diemen, The Netherlands. Email: j.ter.heide@centrum45.nl

First received 1 Jul 2014, final revision 1 Apr 2015, accepted 16 Jun 2015

\section{Funding}

This study was jointly funded by ZonMW, The Netherlands organisation for health research and development, and Foundation Centrum '45, partner in Arq Psychotrauma Expert Group. R.v.d.S was supported by a grant from The Netherlands organization for scientific research: NWO-VENI-451-11-008.

\section{Acknowledgements}

The authors thank all participants and colleagues at Foundation Centrum ' 45 who contributed to this study: Berthold Gersons, Jeroen Knipscheer and Geert Smid who served as advisors: Mariëlle Zondervan-Zwijnenburg who provided statistical assistance; and Paula schnurr and Mirjam Nijdam who commented on earlier versions of this paper.

\section{References}

1 Bogic M, Ajdukovic D, Bremner, S, Franciskovic T, Galeazzi GM, Kucukalic A et al. Factors associated with mental disorders in long-settled war refugees: refugees from the former Yugoslavia in Germany, Italy and the UK. Br J Psychiatry 2012; 200: 216-23.

2 American Psychiatric Association. Diagnostic and Statistical Manual of Mental Disorders (4th edn, text revision) (DSM-IV-TR). APA, 2000.

3 Fazel M, Wheeler J, Danesh J. Prevalence of serious mental disorder in 7000 refugees resettled in western countries: a systematic review. Lancet 2005; 365: 1309-14.

4 Van Ee E, Mooren TM, Kleber RJ. Broken mirrors: shattered relationships in refugee families. In Helping Children Cope with Trauma: Individual, Family and Community Perspectives (eds R Pat-Horenczyk, D Brom, C Chemtob, J Vogel): 146-62. Routledge, 2014.

5 Kivling-Bodén G, Sundborn E. The relationship between post-traumatic symptoms and life in exile in a clinical group of refugees from the former Yugoslavia. Acta Psychiatr Scand 2002; 10: 461-8.

6 Gorst-Unsworth C, Goldenberg E. Psychological sequelae of torture and organised violence suffered by refugees from Iraq. Br J Psychiatry 1998; 172 90-4.

7 National Institute for Clinical Excellence. Post-Traumatic Stress Disorder: The Management of PTSD in Adults and Children in Primary and Secondary Care. Gaskell, 2005.

8 Tol WA, Barbui C, Van Ommeren M. Management of acute stress, PTSD, and bereavement: WHO recommendations. JAMA 2013; 310: 477-8.

9 Cloitre $\mathrm{M}$, Courtois $\mathrm{CA}$, Ford JD, Green $\mathrm{BL}$, Alexander $\mathrm{P}$, Briere J, et al. The ISTSS expert consensus treatment guidelines for complex PTSD in adults.
ISTSS, 2012 (http://www.istss.org/ISTSS_Main/media/Documents/ISTSS Expert-Concesnsus-Guidelines-for-Complex-PTSD-Updated-060315.pdf).

10 Nickerson A, Bryant RA, Silove D, Steel Z. A critical review of psychological treatments of posttraumatic stress disorder in refugees. Clin Psychol Rev 2011; 31: 399-417.

11 Palic S, Elklit A. Psychosocial treatment of posttraumatic stress disorder in adult refugees: a systematic review of prospective treatment outcome studies and a critique. J Affect Disord 2011; 131: 8-23.

12 Robjant K, Fazel M. The emerging evidence for narrative exposure therapy: a review. Clin Psychol Rev 2010; 30: 1030-9.

13 Ter Heide FJJ, Mooren TM, Kleijn W, De Jongh A, Kleber RJ. EMDR versus stabilisation in traumatised asylum seekers and refugees: results of a pilot study. Eur J Psychotraumatol 2011; 2: 5881.

14 Sheehan DV, Lecrubier $\mathrm{Y}$, Harnett-Sheehan $\mathrm{KH}$, Amorim $\mathrm{P}$, Janavs J, Weiller $\mathrm{E}$, et al. The MINI International Neuropsychiatric Interview (M.I.N.I.): the development and validation of a structured diagnostic psychiatric interview for DSM-IV and ICD-10. J Clin Psychiatry 1998; 59: 22-33.

15 Blake DD, Weathers FW, Nagy LM, Kaloupek DG, Gusman FD, Charney DS, et al. The development of a clinician-administered PTSD Scale. J Trauma Stress 1995; 8: 75-90.

16 Mollica RF, Caspi-Yavin Y, Lavelle J, Tor S, Yang T, Chan S, et al. The Harvard trauma questionnaire (HTO): manual; Cambodian, Laotian and Vietnamese versions. Torture 1996; 1 (suppl): 19-34.

17 Mollica RF, wyshak G, de Marnette D, Tu B, Yang T, Khuon F, et al. Hopkins symptom checklist 25 (HSCL-25): manual; Cambodian, Laotian and Vietnamese versions. Torture 1996; 1 (suppl): 35-42.

18 The WHOQOL Group. Development of the World Health Organization WHOQOL-BREF quality of life assessment. Psychol Med 1998; 28 : $551-8$.

19 Schnurr PP, Friedman MJ, Foy DW, Shea MT, Hsieh FY, Lavori PW. Randomized trial of trauma-focused group therapy for posttraumatic stress disorder: results from a Department of Veterans Affairs cooperative study. Arch Gen Psychiatry 2003; 60: 481-9.

20 Shapiro F. Eye Movement Desensitization and Reprocessing: Basic Principles, Protocols and Procedures. Guilford Press, 2001.

21 De Jongh A, Ten Broeke E. Handboek EMDR [EMDR Handbook]. Swets \& Zeitlinger, 2003.

22 Neuner $F$, Kurreck $S$, Ruf $M$, Odenwald $M$, Elbert $T$, Schauer $M$. Can asylum-seekers with posttraumatic stress disorder be successfully treated? A randomized controlled pilot study. Cogn Behav Ther 2010; 39: 81-91.

23 Stenmark H, Catani C, Neuner F, Elbert T, Holen A. Treating PTSD in refugees and asylum seekers within the general health care system: a randomized controlled multicentre study. Behav Res Ther 2013; 51: 641-7.

24 Nijdam MJ, Gersons BPR, Reitsma JB, De Jongh A, Olff M. Brief eclectic psychotherapy $v$. eye movement desensitisation and reprocessing therapy for post-traumatic stress disorder: randomised controlled trial. $\mathrm{Br} \mathrm{J}$ Psychiatry 2012; 200: 224-31.

25 Weathers FW, Ruscio AM, Keane TM. Psychometric properties of nine scoring rules for the Clinician-Administered Posttraumatic Stress Disorder Scale. Assessment 1999; 11: 124-33.

26 Weathers FW, Keane TM, Davidson JRT. Clinician-Adminstered PTSD Scale: a review of the first ten years of research. Depress Anxiety 2001; 13: 132-56.

27 Hinton $\mathrm{DE}$, Chhean D, Pich F, Pollack MH, Orr SP, Pitman RK. Assessment of posttraumatic stress disorder in Cambodian refugees using the ClinicianAdministered PTSD Scale: psychometric properties and symptom severity. J Trauma Stress 2006; 19: 405-9.

28 Hollifield M, Warner TD, Lian N, Krakow B, Jenkins JH, Kesler J, et al. Measuring trauma and health status in refugees: a critical review. JAMA 2002; 288: 611-21.

29 Skevington SM, Lotfy M, O'Connell KA. The World Health Organization's WHOQOL-BREF quality of life assessment: psychometric properties and results of the international field trial. Qual Life Res 2004; 13: 299-310.

30 Kleijn WC, Hovens JE, Rodenburg JJ. Posttraumatic stress symptoms in refugees: assessments with the Harvard Trauma Questionnaire and the Hopkins Symptom Checklist-25 in different languages. Psychol Rep 2001 88: $527-32$.

31 Van de Schoot R, Broere JJ, Perryck KH, Zondervan-Zwijnenburg $M$, Van Loey NE. Analyzing small data sets using Bayesian estimation: the case of posttraumatic stress symptoms following mechanical ventilation in burn survivors. Eur J PSychotraumatol 2015; 6: 25216.

32 Gelman A, Carlin JB, Stern HS, Rubin DG. Bayesian Data Analysis (2nd edn). Chapman \& Hall, 2004 
33 Feingold A. Effect sizes for growth-modeling analysis for controlled clinical trials in the same metric as for classical analysis. Psychol Methods 2009; 14: 43-53.

34 Spinazzola J, Blaustein M, Van der Kolk BA. Posttraumatic stress disorder treatment outcome research: the study of unrepresentative samples? J Traum Stress 2005; 18: 425-36.

35 Cohen J. Statistical Power Analysis for the Behavioral Sciences. Erlbaum, 1988

36 Hembree EA, Foa EB, Dorfan NM, Street GP, Kowalski J, Tu X. Do patients drop out prematurely from exposure therapy for PTSD? J Traum Stress 2003 16: $555-62$

37 Van den Berg DPG, De Bont PAJM, Van der Vleugel BM, De Roos C, De Jongh A, Van Minnen A, et al. Prolonged exposure vs eye movement desensitization and reprocessing vs waiting list for posttraumatic stress disorder in patients with a psychotic disorder: a randomized clinical trial. JAMA Psychiatry 2015; 72: 259-67.

38 Paunovic N, Öst LG. Cognitive-behavior therapy vs exposure therapy in the treatment of PTSD in refugees. Behav Res Ther 2001; 39: 1183-97.

39 Steel Z, Chey T, Silove D, Marnane C, Bryant RA, Van Ommeren M. Association of torture and other potentially traumatic events with mental health outcomes among populations exposed to mass conflict and displacement: a systematic review and meta-analysis. JAMA 2009; 302: 537-49.

40 Benish SG, Quintana S, Wampold BE. Culturally adapted psychotherapy and the legitimacy of myth: a direct-comparison meta-analysis. J Counsel Psychol 2011: 58: 279-89.

Fि EXTRA

\section{extra}

\section{Nise da Silveira (1905-1999), Brazilian psychiatrist and pioneer of rehabilitation psychiatry}

\section{Christopher Kowalski}

'To navigate against the current, these rare qualities are needed: a spirit of adventure, courage, perseverance and passion

- Nise da Silveira

In a time when lobotomies, electroshock and insulin therapies were still the norm in Brazilian psychiatry, Nise da Silveira stood out as a singular voice advocating for a kinder and more relational approach to the treatment of people with mental health conditions. Despite working in an arena dominated by men, she fought passionately against the status quo and was instrumental in establishing occupational therapies as a valid treatment in schizophrenia and other chronic psychiatric conditions in Brazil, changing the way in which rehabilitation was viewed and practised in her native land.

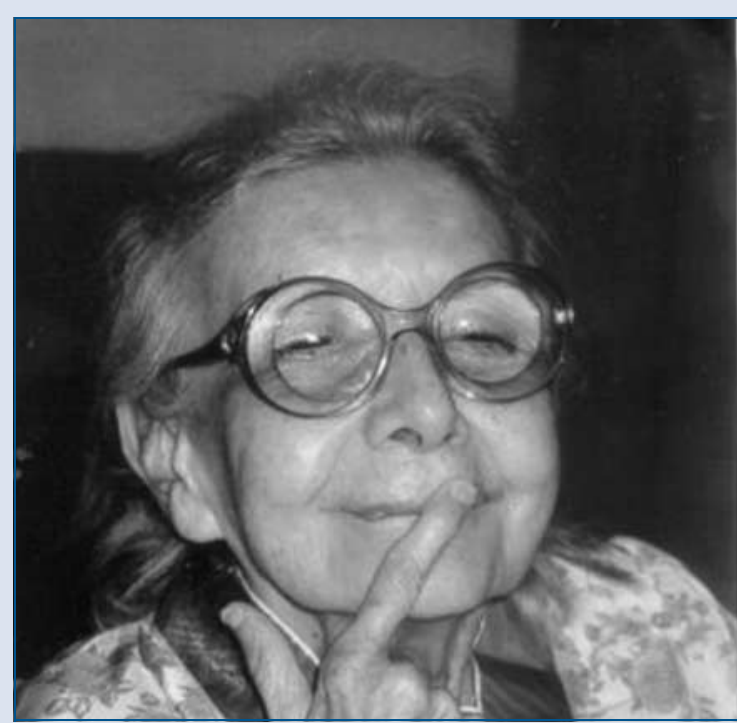

Ever the revolutionary, da Silveira found inspiration in many counter-cultural ideas and ways of thinking, both in the field of psychiatry and outside of it. In 1934, she was imprisoned for 18 months for possessing 'subversive' Marxist literature, having been denounced to the Vargas dictatorship by a nurse with whom she worked. Upon her return to psychiatric practice, da Silveira became increasingly disillusioned with the application of what she saw as aggressive and inhumane treatments in mental health. Inspired by the work of R.D. Laing, David Cooper and Maxwell Jones, she set out to develop new and more compassionate ways of working with those suffering with chronic illness. This led to her establishing the Seção de Terapêutica Ocupacional e Rehabilitação (Department of Occupational Therapy and Rehabilitation) in the Centro Psiquiátrico de Pedro II, Rio de Janeiro, in 1946. Here, she organised workshops for painting and sculpting where patients were encouraged to experiment with new means of emotional expression. A devotee of Carl Gustav Jung, da Silveira applied Jungian ideas to the interpretation of her patients' works in an attempt to develop a deeper and more personal understanding of the individuals in her care and the psychotic processes underpinning their symptoms. The success of this approach was evident early on and many of da Silveira's patients were able to successfully reintegrate back into society.

The works of these patients eventually came to be celebrated in the Museu de Imagens do Inconsciente (Museum of Images of the Unconscious) which da Silveira founded in 1952. The museum has remained in operation to this day and now holds over 350000 works by patients from the 1940s to today.

da Silveira went on to establish the Casa das Palmeiras in 1956, a clinic devoted to the rehabilitation of former patients of psychiatric hospitals which eschewed the institutionalisation and restriction of liberty of its attendees. She pioneered research into the therapeutic potential of animals in recovery, naming them her 'co-therapists', and her work inspired the creation of cultural centres and therapeutic institutions both in Brazil and abroad. She died at the age of 94 , leaving behind a truly unique legacy. Her passion and devotion to the value of emotional connection, creativity and compassion are reminders of the importance of these qualities in both the treatment and rehabilitation of people with mental illness. 